\title{
NY-ESO-1/PRAME/MAGE-A3/WT-1 Peptide Vaccine
}

National Cancer Institute

\section{Source}

National Cancer Institute. NY-ESO-1/PRAME/MAGE-A3/WT-1 Peptide Vaccine. NCI

Thesaurus. Code C127125.

A peptide-based cancer vaccine comprised of synthetic peptides derived from the cancer-testis antigen NY-ESO-1, preferentially expressed antigen in melanoma (PRAME), human melanoma antigen A3 (MAGE-A3) and the human Wilms tumor protein-1 (WT-1), with potential immunostimulating and antineoplastic activities. Upon administration, NYESO-1/PRAME/MAGE-A3/WT-1 peptide vaccine may stimulate the immune system to mount a cytotoxic T-lymphocyte (CTL) response against tumor cells expressing NY-ESO1, PRAME, MAGE-A3 and WT-1, resulting in tumor cell lysis. The NY-ESO-1, PRAME, MAGE-A3 and WT-1 peptides, tumor-associated antigens (TAAs) overexpressed in a variety of cancer cell types, play a key role in tumor cell proliferation. 